\title{
Epidemiologic Response to the First Case of COVID-19 in Nigeria
}

\author{
Tomi Coker ${ }^{1}$, Hakeem Abiola Yusuff ${ }^{1,2, ~ *, ~ O l a d i p o ~ O l a n r e w a j u ~ O g u n b o d e ~}{ }^{3}$, \\ Olawale Sunday Animasaun ${ }^{2,4}$, Adesoji Olatunde Odukoya ${ }^{2,5}$, Olukemi Titilope Olugbade ${ }^{2,6}$, \\ Idris Nasir Abdullahi ${ }^{7}$, Omotayo Yusuff Awere ${ }^{1}$, Gloria Charles Archibong ${ }^{8}$, \\ Adefunke Comfort Adesina ${ }^{9}$, Islamiat Soneye ${ }^{1}$, Amos Akinremi ${ }^{1}$, Adeniyi Hakeem Bisiriyu ${ }^{1,2}$,

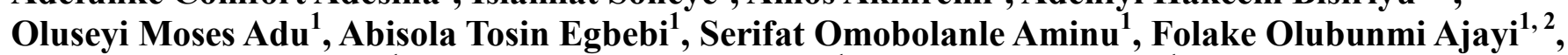 \\ Risquat Oluremi Salako ${ }^{1}$, Festus Olukayode Soyinka ${ }^{1}$, Olukayode Kusimo ${ }^{1}$, \\ Adesanya Ekundayo Ayinde ${ }^{1}$, Sunday Dele-David ${ }^{8}$, Muhammad Shakir Balogun ${ }^{2,10}$ \\ ${ }^{1}$ Ministry of Health, Abeokuta, Nigeria \\ ${ }^{2}$ Nigeria Field Epidemiology and Laboratory Training Programme, Abuja, Nigeria \\ ${ }^{3}$ Nigeria Centre for Disease Control, Abuja, Nigeria \\ ${ }^{4}$ Oyo State Primary Health Care Board, Ibadan, Nigeria \\ ${ }^{5}$ Department of Veterinary and Pest Control Services, Federal Ministry of Agriculture and Rural Development, Abuja, Nigeria \\ ${ }^{6}$ Department of Community Health, Obafemi Awolowo University Teaching Hospitals Complex, Ile-Ife, Nigeria \\ ${ }^{7}$ Department of Medical Laboratory Science, Faculty of Allied Health Sciences, Ahmadu Bello University, Zaria, Nigeria \\ ${ }^{8}$ World Health Organization, Nigeria Office, Abeokuta, Nigeria \\ ${ }^{9}$ Department of Community Medicine and Primary Care, Federal Medical Centre, Abeokuta, Nigeria \\ ${ }^{10}$ Africa Field Epidemiology Network, Abuja, Nigeria
}

\section{Email address:}

hakeemyusuff2019@gmail.com (H. A. Yusuff)

${ }^{*}$ Corresponding author

\section{To cite this article:}

Tomi Coker, Hakeem Abiola Yusuff, Oladipo Olanrewaju Ogunbode, Olawale Sunday Animasaun, Adesoji Olatunde Odukoya, Olukemi Titilope Olugbade, Idris Nasir Abdullahi, Omotayo Yusuff Awere, Gloria Charles Archibong, Adefunke Comfort Adesina, Islamiat Soneye, Amos Akinremi, Adeniyi Hakeem Bisiriyu, Oluseyi Moses Adu, Abisola Tosin Egbebi, Serifat Omobolanle Aminu, Folake Olubunmi Ajayi, Risquat Oluremi Salako, Festus Olukayode Soyinka, Olukayode Kusimo, Adesanya Ekundayo Ayinde, Sunday Dele-David, Muhammad Shakir Balogun. Epidemiologic Response to the First Case of COVID-19 in Nigeria. Central African Journal of Public Health.

Vol. 6, No. 6, 2020, pp. 339-345. doi: 10.11648/j.cajph.20200606.14

Received: October 24, 2020; Accepted: November 16, 2020; Published: December 11, 2020

\begin{abstract}
Coronavirus disease 2019 (COVID-19) caused by severe acute respiratory syndrome coronavirus 2 (SARS-CoV-2) has resulted in an unprecedented global health security challenge, economic downturns and psychosocial disruptions. First reported in Wuhan, China, the epidemic swiftly spread to over 200 countries and territories. In Nigeria, the first confirmed COVID-19 case was imported via air travel on $24^{\text {th }}$ of February 2020 and Confirmed on $27^{\text {th }}$ of February 2020. Minimal public health surveillance activities were initiated before then. In response to the confirmation of COVID-19, incident management activities, epidemiologic surveillance, contact tracing, infection, prevention and control trainings, COVID-19 testing and activation of isolation and treatment centers were immediately implemented in Ogun State. The objective of the outbreak investigation in Ogun State was to determine the magnitude of the outbreak, characterize the outbreak, and implement disease detection and response activities. This report documents and reflects on the steps taken to mitigate the initial spread of COVID19 and the outbreak response activities in Ogun State, Nigeria. Adequate risk communication, robust surveillance, effective contact tracing and isolation of primary contacts of the first confirmed case in Nigeria as well as implementation of infection, prevention and control measures were pivotal tools that helped to curtail the initial spread of the infection. Operative collaboration between all stakeholders involved in response and surveillance to emerging dangers is necessary to detect cases early and to implement adequate preventive and control measures.
\end{abstract}


Keywords: Epidemiologic Response, Index Case, SARS-CoV-2, Surveillance, Nigeria

\section{Introduction}

The World Health Organization (WHO) Country Office in China on the 31st of December, 2019 was informed of reports about pneumonia cases of unknown origin in Wuhan City, Hubei Province. Subsequently, the novel coronavirus causing the cluster of respiratory disorders, initially called 2019-nCoV was isolated from human throat swab samples and later named Severe Acute Respiratory Syndrome Coronavirus 2 (SARS-CoV2) on the 11th of February 2020, whereas the disease was referred to as Coronavirus Disease 2019 [1].

By 30th of January 2020, WHO declared the COVID-19 outbreak a public health emergency of international concern, because it has caused an unprecedented pandemic that has affected most countries and territories of the globe. As of 7:30 pm CEST, 9th of August 2020, there have been 19,929,904 globally confirmed cases of COVID-19 and 731,846 COVID-19 attributed deaths [1].

The pandemic spreads to Africa, with the index case confirmed in Egypt on $14^{\text {th }}$ of February 2020, since then, 47 countries in the region have recorded 881,242 confirmed cases and 16,359 deaths as at 9th of August 2020 [2]. The first confirmed case of COVID-19 in Nigeria was an Italian national who arrived through the Murtala Muhammed International Airport (MMIA) Lagos on the $24^{\text {th }}$ of February 2020 from Milan and subsequently developed flu-like symptoms while at work in a company site in Ewekoro Local Government Area, Ogun State, South West Nigeria, about 48 hours after his arrival.

Following high index of suspicion by the attending physician at the facility in Ewekoro. Suspecting that he met the case definition for COVID-19, based on his travel history, he was isolated immediately and thereafter moved to the Infectious Disease Hospital in Yaba, Lagos State, which has one of the Nigeria Centre for Disease Control (NCDC)'s certified laboratories network for COVID-19 testing. The Italian national eventually turned out Reverse Transcriptase Polymerase Chain Reaction (RT-PCR) positive for SARSCOV-2 on Thursday 27th of February 2020. In compliance with International Health Regulations, the Ogun State government immediately notified the NCDC and the Federal Ministry of Health (FMOH) with announcement of the finding on Friday 28th of February 2020.

The State Rapid Response Team (RRT) swung into action, signalling series of events that constituted a coordinated outbreak response. Following a technical briefing at the State with representatives from the $\mathrm{FMOH}, \mathrm{NCDC}$, and $\mathrm{WHO}$, an Emergency Operations Centre (EOC) with an Incident Manager and an eight-pillar team inaugurated and activated. Aggressive sensitization with risk communication, community engagement, the involvement of the public, and all stakeholders via face-to-face meetings, electronic, print and social media communication initiated. Facility assessment and decontamination, line listing and contact tracing of every individual who had suspicion of contact with the index case irrespective of their level of contact, and subsequent 14 days quarantine observed. Sensitization of staff members in the company, where the index case works and healthcare staff of the company's health facility were trained on Infection Prevention and Control (IPC) and standard precaution measures. 217 contacts across the nation were tracked and monitored for the development of any of the symptoms associated with COVID-19.

The President, at the national level constituted a COVID19 Presidential Task Force saddled with the responsibilities of coordinating the national response, releasing public health advisories, advocacy, communication, and press briefings.

The objectives of this outbreak response were to determine the magnitude of the outbreak, characterize it, and implement epidemiologic surveillance and contact tracing activities to curtail the outbreak. This report reflects on the successful steps taken to mitigate the initial spread of COVID-19 within Ogun State and in Nigeria. It serves as a guide on the role of surveillance system in the detection of priority diseases and events of public health importance. Especially in disease determinants: causes, and risk factors, in the impact of interventions, in the facilitation of evidence-based response, in the evaluation of the impact of a pandemic on health-care systems and in monitoring long term epidemiologic trends and evolution of infections such as the COVID- 19.

As of 9th August 2020, 24 weeks after the index case confirmation, there were 46,577 confirmed COVID-19 cases in Nigeria and out of this, 1,469 are in Ogun with a case fatality rate of $1.6 \%$ [3].

Currently, securing human health, minimizing COVID-19 associated morbidity and mortality is a national priority, consequently, public health authorities and stakeholders need to have in place structures and strategies for coordinated surveillance, case detection, contact tracing systems; and infection prevention, control plans, and protocols [4].

The Integrated Disease Surveillance and Response (IDSR) System seeks to ensure that an effective and functional IDSR system is available at each level of the health system, from health facilities to Local Government Areas, States and at the National level [5]. The IDSR system focuses on the LGA level where the information generated used for timely action consequently leading to a reduction of morbidity, disability, and mortality [5].

Ogun State has a functional surveillance system established in line with the National IDSR core functions and has been responding to outbreaks such as Viral Hemorrhagic Fever (VHFs), measles, rubella, cholera and yellow fever [6]. The evaluation of state surveillance system for tuberculosis and other respiratory diseases (like pneumonia) and reports showed it is sensitive and reliable [6]. Based on the existing 
structure, surveillance of COVID-19 cases was implemented in the state from 28th of February 2020 to identify possible imported cases early and to prevent community transmission. Based on the strong surveillance network in the state, contact tracing of the index case was initiated; 40 contacts were line listed and quarantined for 14 days and observed for the possible development of symptoms.

\section{Outbreak Setting}

The first case of COVID-19 in Nigeria was reported in Ewekoro Local Government Area (LGA) on 27th of February 2020. Ewekoro is one of the 20 LGAs in Ogun State, Nigeria. Its headquarters is in Itori town at $6^{\circ} 56^{\prime} 00^{\prime \prime} \mathrm{N}$ $3^{\circ} 13^{\prime} 00^{\prime \prime}$. Ewekoro LGA covers a landmass of about 1,685 $\mathrm{km}^{2}$. The LGA shares borders with Ifo in the South, Yewa North and South LGAs in the West, Abeokuta North LGA in the north, and the East by Obafemi Owode LG. Towns and villages that constitute Ewekoro LGA include Asipa, Ikereku, Ilawo, Itori Alase, Lambo, Pataleri, Ojo, and Ika. The population of Ewekoro LGA is estimated at 86,895 (projected population from 2006 population census) with most of the inhabitants being members of the Egba subdivision of the Yoruba tribe. The Yoruba language and English are widely spoken in the area. Ewekoro LGA has large deposits of limestone and has the presence of a renowned multi-national Cement Factory.

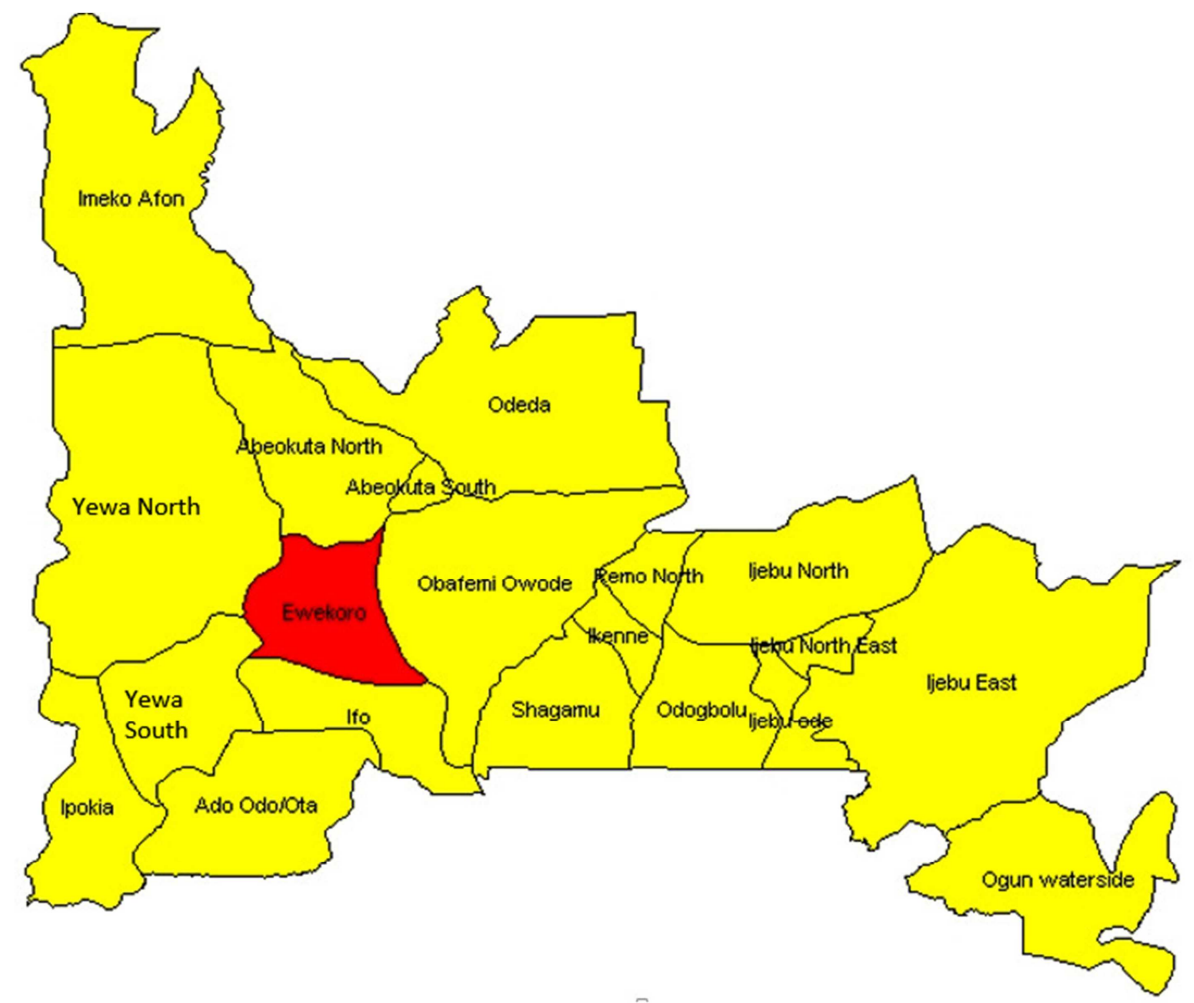

Figure 1. Map of Ogun State Showing the 20 Local Governments Areas with the LGA where the index case of COVID-19 was identified in red.

Case definition for covid-19 in Nigeria

Case definition in Nigeria for COVID-19 has been evolving following global realities and in-country findings. As at the time the first case was reported in Nigeria Version 1 in use stated the following:

Case definition for new coronavirus disease (version 1)

Suspected Case: Any person with acute respiratory illness, (including severely ill patients who have been hospitalized) presenting with fever, cough, difficulty breathing AND who within 14 days before the onset of illness has any one of the following exposures

(1) History of travel to China 14 days before symptoms onset OR (2) Close contact with a confirmed case of SARSCoV-2 infection OR

(3) Exposure to healthcare facility in a country where hospital-associated SARS-CoV-2 infections have been reported

Probable case: A suspect case for whom testing for SARS-
CoV-2 is inconclusive or for whom testing was positive on a pan-coronavirus assay

Confirmed case: Any person with laboratory confirmation of SARS-CoV-2 infection with or without signs and symptoms

This has been continuously revised and we now have versions 2, 3, 4 and 5 [3].

\section{Case Detection and Investigation}

Following confirmation from the laboratory, the Ogun State Ministry of Health was notified through the State Epidemiologist. Initiation of Series of activities by the state RRT followed. This signals series of events beginning with an emergency meeting with relevant stakeholders (NCDC, WHO, and AFENET). Ogun State Emergency Operation Center (OGEOC) was activated to respond to the outbreak; 
visitation to the facility (where infected areas were cordoned off and decontaminated), line listing and contact tracing of every individual who had been suspected of having contact with the index case irrespective of their level of contact and quarantine.

Sensitization of staff of the facility and staff of the medical center on Infection Prevention and Control (IPC). Intensive risk communication and sensitization on IPC was conducted in all the communities in Ewekoro LGA with the distribution of Information Education and Communication (IEC) materials on frequently asked questions (FAQs), hand and respiratory hygiene, physical distancing, avoiding mass gatherings, case definitions, 5 moments of hand hygiene, and Ogun State emergency toll-free hotline numbers.

The 40 contacts identified followed quarantine protocol strictly in accommodation provided by the company. Followup and daily temperature measurement of all quarantined contacts conducted from day 3 to day 14. All the primary contacts of the index case followed quarantine protocol for 14 days. They were monitored daily, all other contacts in other states were asked to practice self-quarantine and were monitored for compliance daily, they were told to wash their hands frequently, to wear masks when in the same room as other household members, not to share personal items (e.g., towels, drinking cups, or cutlery), and to sleep in separate rooms. Temperature measurement reading conducted twice daily.

Data collection tools used for case investigation, contact tracing, monitoring, and follow-up were Case Investigation Form (CIF) A1, Contact Investigation Form B1, IDSR 001C Line list form, Daily monitoring chart.

Lessons learnt from other countries' experience and publications as well as the recommendation of the United State Centers for Disease Control, which stipulates testing of all close contacts of confirmed or probable COVID-19 patients [7], led the response team to collect samples from the 40 contacts who were asymptomatic for COVID-19 as at the quarantine period.

On the 5th of March, 2020 (day 7 of quarantine), nasal and throat swabs were collected from each of the 40 contacts and transported in a Viral Transport Medium (VTM) to Lagos Virology Lab for COVID-19 testing using Reverse Transcriptase Polymerase Chain Reaction (RT-PCR) method. One came out positive for COVID-19 on 7th March, 2020, makes the second confirmed case in Nigeria. He was moved from his isolation room to IDH, Lagos on 8th of March, 2020 for management. No contact was associated with him due to quarantine.

Exit screening exercise performed on 9th of March, 2020 (day 12 of quarantine). Two nasal and two throat swabs each collected from the remaining 39 contacts and transported simultaneously to Lagos Virology Lab and the NCDC National Reference Laboratory (NRL) as a way of ensuring quality control for COVID-19 testing and seeking a second opinion using RT-PCR method at both labs. All the samples came out Negative for COVID-19 and since they have all completed their 14 days isolation and had no symptoms, were all discharged from isolation to integrate back to the community on the 12th March 2020.

The Nigeria COVID-19 index case after successful management and tested negative twice to SARS-COV 2 was discharged from the IDH, Lagos on 20th of March, 2020.

\section{Ethical Consideration}

Ethical approval with Ethical Approval number HPRS/381/344 was obtained from the Ogun State Ministry of Health Ethical Research Committee. Authorized personnel anonymized confidentiality of data collected from all contacts.

\section{Results}

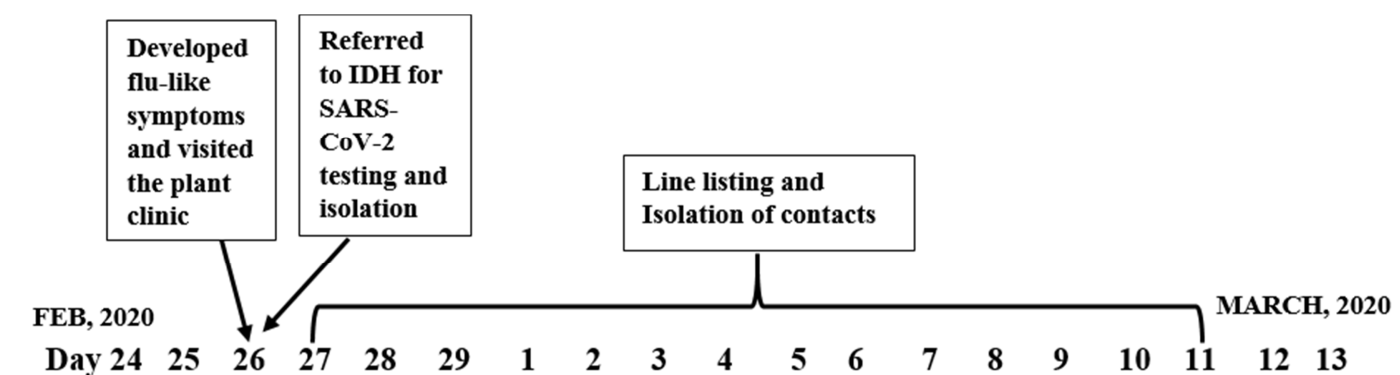

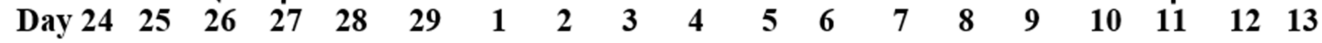

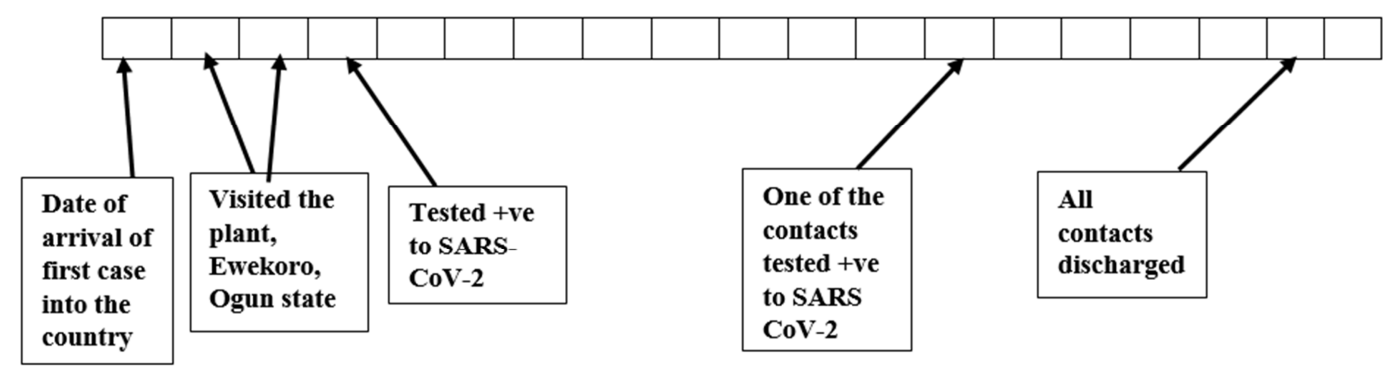

Figure 2. Timeline of activities. 


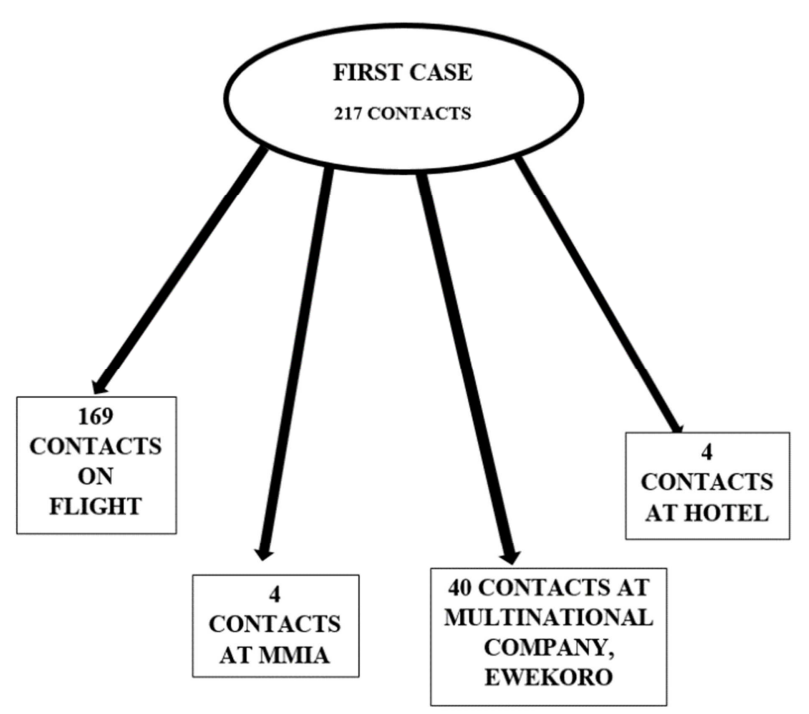

Figure 3. Distribution of Contacts according to Location of Exposure, Ogun State, Nigeria.

\subsection{Distribution of Contacts}

We identified 217 contacts linked to the first case in Nigeria; 169 contacts on the flight from Milan, Italy, four at the MMIA Lagos, four at the hotel in Lagos, and 40 persons at the company in Ewekoro, Ogun State.

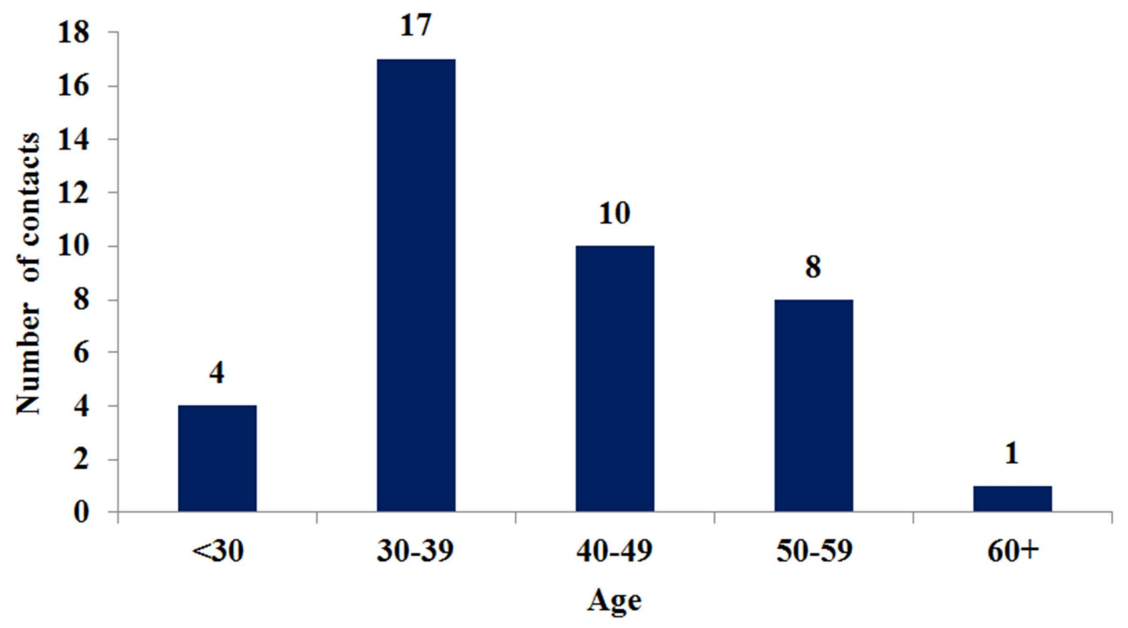

Figure 4. Age Distribution of Contacts of COVID-19 Index Case, Ogun State, Nigeria.

\section{Discussion}

This report describes the response to the first case of COVID-19 in Nigeria, epidemiological characterization of the case and contacts (in terms of time, place and person), highlighting the surveillance activities and outbreak investigation outcomes in Ogun State where the index case was identified [8]. The report of the first imported and positive contact emphasizes the role of having a high index of suspicion and early isolation in containing the spread of COVID-19 and epidemic prone infectious diseases, given that there was a positive contact case in the State, despite all the contacts investigated being asymptomatic.
Forty-five contacts linked to the case had traveled out of Nigeria to their different destinations at the time of confirmation. Within the country, 171 contacts were available for contact tracing; 69 contacts in Lagos State, 40 in Ogun State, and 52 across other states (Akwa-Ibom, Anambra, Bayelsa, Cross River, Edo, Imo, Osun, Oyo, and Rivers). All contacts observed 14 days followed up. In this report, we focus on contacts identified within Ogun State.

\subsection{Contacts in Ogun State}

A total of 40 contacts were identified and followed up in the company's guest house at Ewekoro which was used as a temporary isolation center in Ogun State from Day 2 or Day 3 of exposure (29th of February 2020) based on their last day of contact. All contacts observed quarantined in a separate room. Nine were female (22.5\%) and 31 (77.5\%) male, and comprised of $7(17.5 \%)$ health care workers and $33(82.5 \%)$ non-healthcare workers.

All the 39 contacts and the negative case were discharged on the 12th of March, 2020 after being given a sensitization, and orientation, post-discharge health education, and a discharge certificate.
Although the case definition for COVID -19 is updated periodically, the case definition as at the time of the index case stipulated travelers from China. The index case however did not arrive from China nor did he travel to China. As found in other countries, the index case of COVID-19 in Nigeria was an imported case; who travelled from Italy and not China as the case might be in other country.

Global awareness about the pandemic by WHO and incountry sensitization by the NCDC were beneficial in raising the index of suspicion among healthcare workers in Ogun State, this was evident when the attending physician assessed the index case as a suspected case of COVID-19. The case definition stating the symptoms to anticipate developed by 
the NCDC played a crucial role in screening and triaging.

Field investigations importance document by center for disease control shows highlights [9]. Reporting "investigations often lead to the identification of additional unreported or unrecognized ill persons who might otherwise continue to spread infection to others". It can thus be rationally assumed that the source of infection in the only contact that tested positive out of the forty contacts investigated in the state was a close contact with the index case as he spent more time together with him throughout his approximately 48 hours stay before symptoms onset. The contact that tested positive within seven days of exposure shows consistent with the reported incubation period of 2 to 14 for COVID-19. This is also similar to the account of the first two cases of coronavirus disease in the United Kingdom where the period between exposure and infection was estimated to be 48 hours implying that the disease was highly infectious [10]. Moreover, male gender and middle age (47 years) of the positive contact case was consistent with a study in the United Kingdom that found association between a positive test for COVID-19 in primary care and sociodemographic factors like male gender and age older than 40 years [11]. The experience of Ogun State Government of Nigeria with the first case and the contacts traced was however different from that of the United Kingdom (UK) as regards distribution of contacts of the index case, who were work contacts and not household contacts; they were all asymptomatic with one person testing positive. Other studies have shown disease transmission to be commoner amongst household contacts [10]. However, they suggested investigation of the serological responses in asymptomatic contacts to ascertain evidence of subclinical infection. This current report may be limited in its generalization as it only gave account of the contact tracing, testing and management of the forty contacts identified within Ogun State out of the total two hundred and seventeen contacts linked to the first case in Nigeria. The suggestion of on-going community transmission of COVID-19 indicated by the pattern of the epidemic curve [12]. (Bamidele and Daniel, 2020) highlights the need to strengthen epidemiologic response including the six major tasks of epidemiology in public health practice reported by the United States Centers for Disease Control; and Prevention [9]. as "public health surveillance, field investigation, analytic studies, evaluation, linkages, and policy development".

Ogun State Government coordinated response to the first case of COVID-19 in Nigeria played a major role in delaying community transmission and limiting population exposure. The only gap of surveillance system observed, during the course of the case detection and response in the state was the breach of reporting protocol; the attending physician notified the closest NCDC laboratory and instructed the suspected case tested for COVID-19 without following the surveillance system protocol on ground in the state. Protocol is first notification of the LGA Disease Surveillance and Notification Officer's (DSNO) and State Epidemiologist, before other response activities commence.
Testing all the contacts of the index case of COVID-19 in Nigeria who at the time were asymptomatic was not in line with the guidelines of Africa $\mathrm{CDC}$ who advised that contacts could undergo COVID-19 test when they have any indication of COVID-19 symptoms and categorically stated that contacts without symptoms might not be tested. Africa CDC advised that testing contacts without symptoms drain resources. A negative test result or absence of symptoms will not change the advice given for a contact to stay at home for 14 days due to an asymptomatic early phase of infection, not detectable by the PCR test. A negative test may give false reassurance and encourage a person to reduce adherence to quarantine and individual infection prevention measures [13]. However, the response showed that testing the contacts of the index case during the period of quarantine enabled the State RRT detect the second case for prompt management at the designated treatment center hence breaking the chain of transmission [14].

\section{Conclusion}

The COVID-19 pandemic has affected all structures of the global economy and the health care systems worldwide, and calls for a need to capture the trend of the events at the dawn of the outbreak in Nigeria [15]. The rapid and collaborative response to the first COVID-19 confirmed case in Nigeria is an indicator of the coordinated level of preparedness in the country and a marker of political will, commitment and evidence that the Nigerian healthcare system was prepared to respond to an emerging disease threat of such magnitude.

Surveillance of the contacts of the COVID-19 index case in Nigeria and the implementation of control measures, including prompt testing and quarantine of all the contacts, decreased the risk of human-to-human transmission originating from the imported case, this subsequently delayed propagation of the virus in the general population [14].

\section{Conflict of Interest}

The authors declare that they have no competing interests.

\section{References}

[1] Electronics, World Health Organization (WHO) (2020) Retrieved June 2020 from https://www.who.int/emergencies/diseases/novel-coronavirus2019.

[2] Electronics, Africa Centers for Disease Control and Prevention (ACDC) (2020A) Retrieved June 2020 from https://africacdc.org/covid-19/.

[3] Electronics, Nigeria Centre for Disease Control (NCDC) (2020) Retrieved March 2020 from https://covid19.ncdc.gov.ng/.

[4] Elvis E. Isere. (2015). An overview of disease surveillance and notification system in Nigeria and the roles of clinicians in disease outbreak prevention and control. Nigerian Medical Journals 56 (3): 161-168. doi: 10.4103/0300-1652.160347. 
[5] Electronics, Nigeria Centre for Disease Control (NCDC) (2020) Retrieved March 2020 from https://ncdc.gov.ng/diseases/guidelines.

[6] Abdulraheem I. S. (2004). Disease notification among physicians in a Nigerian Tertiary Health Institution. African Journals Online (AJOL) Vol. 45, No. 6 (2004); pp. 111-115. DOI: $10.4314 /$ nmp.v45i6.28715.

[7] Electronics, Centre for Disease Control (CDC) (2020) Retrieved March 2020 from https://www.cdc.gov/coronavirus/2019-ncov/php/contacttracing/contact-tracing-plan/contact-tracing.html.

[8] Sibylle B. S. (2020) "First cases of coronavirus disease 2019 (Covid-19) in France: surveillance, investigations and control measures, January 2020" Euro Surveillance Journal, 25 (6): 17 https://doi.org/10.2807/1560-7917.ES.2020.25.6.2000094.

[9] Electronics, CDC (2012) "Principles of Epidemiology in Public Health Practice, Third Edition, An Introduction to Applied Epidemiology and Biostatistics", Reviewed 18 May 2012, Accessed June 2020 at https://www.cdc.gov/csels/dsepd/ss1978/lesson1/section4.html.

[10] Patrick J. Lillie, (2020) "Novel Coronavirus Disease (Covid19): The First Two Patients in the UK With Person to Person Transmission", Journal of Infection, 80 (5): 578-606. doi: 10.1016/j.jinf.2020.02.020.
[11] Simon de Lusignan, (2020) "Risk factors for SARS-CoV-2 among patients in the Oxford Royal College of General Practitioners Research and Surveillance Centre primary care network: a cross-sectional study", Lancet Infect Disease 20 (9): 1034-1042. doi: 10.1016/S1473-3099(20)30371-6.

[12] Bamidele JO and Daniel OJ (2020) "Epidemiology of Coronavirus Disease (COVID-19) in Nigeria", Annals of Health Research, 6 (2): 125-132. DOI: https://doi.org/10.30442/ahr.0602-01-74.

[13] Electronics, Africa Centers for Disease Control and Prevention (ACDC) (2020B) Retrieved March 2020 from https://au.int/sites/default/files/documents/38351-doc guidance_on_contact_tracing_for_covid19_pandemic_eng.pdf.

[14] Electronics, World Health Organization. Statement on the second meeting of the International Health Regulations (2005) Emergency Committee regarding the outbreak of novel coronavirus (2019-nCoV). Retrieved November 2020 from https://www.who.int/news/item/30-01-2020-statement-on-thesecond-meeting-of-the-international-health-regulations(2005)-emergency-committee-regarding-the-outbreak-ofnovel-coronavirus-(2019-ncov).

[15] Electronics, Jerome A. S, Sunita VS Bandewar and Elizabeth A. B "The impact of the COVID-19 pandemic response on other health research. Retrieved November 2020 from https://www.who.int/bulletin/volumes/98/9/20-257485/en/. 